Forward Pharma, Genentech, GlaxoSmithKline, Janssen, Kyowa Hako Kirin, Kythera, Leo Pharma, Merck and Co, Merck-Serono, Novartis, Pfizer, Regeneron, Rigel, Roche, Sanofi-Genzyme, Takeda, UCB, Valeant, Xenon, and Xoma., M. Gooderham Grant/research support from: AbbVie, Amgen, Boehringer Ingelheim, Celgene, Coherus, Dermira, Galderma, GSK, Jansen, Leo Pharma, Lilly Medimmune, Merck Serono, Novartis, Regeneron, Roche, Sanofi Genzyme, Takeda, UCB, Pfizer, and Valeant., Consultant for: AbbVie, Amgen, Boehringer Ingelheim, Celgene, Coherus, Dermira, Galderma, GSK, Jansen, Leo Pharma, Lilly Medimmune, Merck Serono, Novartis, Regeneron, Roche, Sanofi Genzyme, Takeda, UCB, Pfizer, and Valeant., Speakers bureau: AbbVie, Amgen, Boehringer Ingelheim, Celgene, Coherus, Dermira, Galderma, GSK, Jansen, Leo Pharma, Lilly Medimmune, Merck Serono, Novartis, Regeneron, Roche, Sanofi Genzyme, Takeda, UCB, Pfizer, and Valeant., Y. Zhao Employee of: Sun Pharmaceuticals, S. Lowry Employee of: Sun Pharmaceuticals, A. Mendelsohn Employee of: Sun Pharmaceuticals, J. Parno Employee of: Sun Pharmaceuticals, Q. Li Employee of: Merck and Co., Inc., C. Rosa Employee of: Merck and Co., Inc., K. Reich Grant/research support from: Abbvie, Amgen, Biogen-Idec, Celgene, Centocor, Covagen, Forward Pharma, GlaxoSmithKline, Janssen-Cilag, Leo, Lilly, Medac, Merck and Co, Novartis, Pfizer, Vertex, and Takeda., Consultant for: Abbvie, Amgen, Biogen-Idec, Celgene, Centocor, Covagen, Forward Pharma, GlaxoSmithKline, Janssen-Cilag, Leo, Lilly, Medac, Merck and Co, Novartis, Pfizer, Vertex, and Takeda., Speakers bureau: Abbvie, Amgen, Biogen-Idec, Celgene, Centocor, Covagen, Forward Pharma, GlaxoSmithKline, Janssen-Cilag, Leo, Lilly, Medac, Merck and Co, Novartis, Pfizer, Vertex, and Takeda.

DOI: 10.1136/annrheumdis-2018-eular.2983

\section{AB0956 LEUCINE-RICH ALPHA-2 GLYCOPROTEINIS A PREDICTABLE BIOMARKER FOR THERAPEUTIC RESPONSE AND CLINICAL RELAPSE TO BIOLOGICS, BUT NOT TO APREMILAST IN PATIENTS WITH PSORIASIS}

Y. Shibata ${ }^{1}$, S. Serada ${ }^{2}$, M. Fujimoto ${ }^{2}$, H. Nakajima ${ }^{1}$, S. Sano ${ }^{1}$, T. Naka ${ }^{2,3}$. ${ }^{1}$ Dermatology; ${ }^{2}$ Center for Intractable Immune Disease, Kochi Medical School, Kochi; ${ }^{3}$ Laboratory of Immune Signal, National Institutes of Biomedical Innovation, Health and Nutrition, Osaka, Japan

Background: Leucine-rich alpha-2 glycoprotein (LRG) is a $50 \mathrm{kDa}$ protein produced by hepatocytes, endothelial cells, neutrophils and macrophages, and it was identified as an inflammatory biomarker that correlates with the disease activity of autoimmune diseases such as inflammatory bowel disease and rheumatoid arthritis. We recently reported that LRG could also be a biomarker of psoriasis, which correlated with the clinical severity scores such as Psoriais Area and Severity Index (PASI), Disease Activity Score 28 (DAS-28) and Bath Ankylosing Spondylitis Disease Activity Index (BASDAI) more closely than common inflammation markers like C-reactive protein (CRP) and erythrocyte sedimentation rate (ESR). The biologics are innovative therapies for psoriasis, however they can cause side effects and increase medical cost. Recently, apremilast, a small molecure inhibitor of phosphodiesterase 4 , was approved for treatment of psoriasis in Japan, yet the precise mechanism remains unknown.

Objectives: To explore the eligibility of serum LRG for a biomarker to monitor the responses to biologics and apremilast in psoriasis.

Methods: Antibodies to TNF-alpha, IL-12/IL-23p40, IL-17A and IL-17 receptor A and apremilast were administered in patients with psoriasis vulgaris and psoriatic arthritis ( $n=15,6,14,4$ and 8 respectively). Serum LRG levels were measured by enzyme-linked immunosorbent assay. Serum CRP and ESR, and PASI, DAS-28 and BASDAI were also recorded.

Results: Serum LRG decreased along with the improvement of clinical scores after the administration of biologics, and reflected the change of scores more accurately than CRP and ESR. Furthermore, the LRG levels preceded the changes of clinical symptoms and predicted both primary and secondary treatment failure at the early time point, allowing us to determine if we should increase the doses, discontinue or switch to another drug. In some patients with PASI clear, complete regression of eruption, after biologics, serum LRG rerose from the baseline while their PASI scores remained stable; however, they later relapsed. On the other hand, LRG did not reflect the therapeutic effectiveness of apremilast.

Conclusions: Serum LRG in psoriasis patients would be a sensitive biomarker for predicting the effectiveness and treatment failure of biologics, but not of apremilast. Monitoring LRG levels may enable us to decide the timing of bio-attenuation and to detect the relapse after discontinuation of biologics.

\section{REFERENCE:}

[1] Leucine-rich $\alpha-2$ glycoprotein is an innovative biomarker for psoriasis. J Dermatol Sci. 2017 May;86(2):170-174

Disclosure of Interest: Y. Shibata Grant/research support from: AbbVie GK, S. Serada: None declared, M. Fujimoto: None declared, H. Nakajima Grant/research support from: AbbVie GK, S. Sano Grant/research support from: AbbVie GK, T. Naka: None declared

DOI: 10.1136/annrheumdis-2018-eular.1660

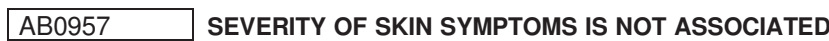 WITH MUSCULOSKELETAL MANIFESTATIONS IN PATIENTS WITH PSORIASIS}

Y. Yamada ${ }^{1,2}$, K. Inui ${ }^{1}$, T. Okano ${ }^{1}$, Y. Sugioka ${ }^{3}$, H. Yoshimura ${ }^{4}$, K. Mamoto $^{1}$ T. Koike ${ }^{5}$, M. Tada ${ }^{6}$, C. Tateishi ${ }^{7}$, E. Yonezawa ${ }^{7}$, A. Natsumi ${ }^{7}$, Y. Fukunaga ${ }^{7}$, D. Tsuruta ${ }^{7}$, H. Nakamura ${ }^{1} .{ }^{1}$ Department of orthopedics surgery, OSAKA CITY UNIVERSITY; ${ }^{2}$ Department of orthopedics surgery, Yodogawa Christian Hospital; ${ }^{3}$ Center for Senile Degenerative Disorders (CSDD), OSAKA CITY UNIVERSITY, Osaka City; ${ }^{4}$ Shirahama Foundation for Health and Welfare; ${ }^{5}$ Search Institute for Bone and Arthritis Disease (SINBAD), Shirahama Foundation for Health and Welfare, Wakayama; ${ }^{6}$ Department of orthopedics surgery, Osaka City General Hospital; ${ }^{7}$ Department of Dermatology, OSAKA CITY UNIVERSITY, Osaka City, Japan

Background: Among patients with psoriasis, risk factors for developing musculoskeletal manifestations, known as psoriatic arthritis (PsA), are not recognised well.

Objectives: The aim of this study is to clarify the relationship between severity of skin disease and arthritis.

Methods: Psoriasis patients referred from dermatologists for assessment of musculoskeletal manifestations between June 2015 and July 2017 were enrolled. Their age, comorbidity, disease duration and treatment were collected. Presence of inflammatory back pain, sacroiliac joint tenderness or enthesitis were examined. Severity of skin symptoms were evaluated by dermatologists in Psoriasis area and severity index (PASI). Psoriatic arthritis screening and evaluation (PASE) and disease activity score (DAS28 ESR) were also evaluated. PsA was diagnosed by The Classification for Psoriatic Arthritis (CASPAR) criteria assisted with musculoskeletal ultrasound examination.

Results: Among 107 patients with psoriasis referred from dermatologists during designated period, 63 patients were diagnosed as PsA. These PsA patients were compared with 44 patients who had no arthritis ( $\mathrm{PsO}$ ). Multiple logistic regression analysis showed neither of age, sex, PASI, disease duration, rheumatoid factor (RF), CRP or Matrix Metalloproteinase-3 (MMP3) had no association with presence of PsA (table 1). Among 63 patients with PsA, those using NSAIDs $(p=0.028)$, those with inflammatory back pain $(p=0.002)$ and male patients $(\mathrm{p}=0.017)$ had significantly high PASI. PASI significantly correlated with age (Spearman's correlation coefficient $R=-0.303$ : $p=0.016)$, body height $(R=0.301$ : $\mathrm{p}=0.019)$ and weight $(\mathrm{R}=0.383: \mathrm{p}=0.002)$, but not with DAS28 $E S R, M M P 3$ or disease duration (table 2 ).

Table 1 multiple logistic regression analysis for presence of PsA

\begin{tabular}{lccc}
\hline variables & OR & $95 \% \mathrm{Cl}$ & $\begin{array}{c}\mathrm{P} \\
\text { value }\end{array}$ \\
\hline age & 0.99 & $0.95-1.03$ & 0.538 \\
sex & 2.13 & $0.66-6.90$ & 0.207 \\
PASI & 0.96 & $0.89-1.03$ & 0.258 \\
disease & 0.99 & $0.96-1.04$ & 0.847 \\
duration & & $0.99-1.01$ & 0.408 \\
RF & 0.99 & $0.76-$ & 0.125 \\
CRP & 2.76 & 10.10 & 0.271 \\
& & $1.00-1.01$ & \\
MMP3 & 1 &
\end{tabular}

Abstract AB0957 - Table 2. Correlations with PASI in patients with PSA (Spearman's correlation)

\begin{tabular}{lcc}
\hline variables & $\mathrm{R}$ & $\begin{array}{c}\mathrm{P} \\
\text { value }\end{array}$ \\
\hline age & -0.303 & 0.016 \\
body height & 0.301 & 0.019 \\
body weight & 0.383 & 0.002 \\
disease & 0.134 & 0.311 \\
duration & & \\
PASE & 0.088 & 0.504 \\
DAS28 ESR & -0.268 & 0.110 \\
RF & 0.152 & 0.273 \\
CRP & 0.211 & 0.105 \\
MMP3 & 0.133 & 0.310 \\
\hline
\end{tabular}

Conclusions: PASI did not associate with presence of arthritis. Furthermore, even DAS28 ESR, reflecting musculoskeletal manifestations, or disease duration did not correlated with PASI among patients with PsA. These indicates severity of skin symptoms is not associated with musculoskeletal manifestations in patients with psoriasis.

Disclosure of Interest: None declared

DOI: 10.1136/annrheumdis-2018-eular.3516 


\section{Osteoarthritis}

\section{AB0958 KNEE PAIN IN OSTEOARTHRITIS: CORRELATION WITH SONOGRAPHIC FINDINGS}

A. Radwan, A. Radwan, M. Khalifa, A.M. Elsaman. Rheumatology and Rehabilitation, Sohag University, Sohag, Egypt

Background: Osteoarthritis $(\mathrm{OA})$ is a common joint disorder, with the knee being one of the most frequently involved sites. Knee OA causes pain and stiffness and can lead to considerable disability and consequently to a reduced quality of life (Wideman et al. 2014). The level of radiographic knee OA is, at most, moderately associated with the level of pain. Therefore, it is unlikely that pain is predominantly caused by only bone and cartilage pathology. Mechanical, structural, inflammatory, bone-related, neurological and psychological factors play a role in the process that results in painful knee OA (Wenger et al. 2013).

As $\mathrm{OA}$ is a disease of the entire joint that is characterised by cartilage breakdown, subchondral bone alterations and formation of osteophytes, as well as soft tissue abnormalities including meniscal degeneration, bursitis, tendonitis, Baker's cyst and synovial inflammation; information about these soft tissue structures might provide more insight into their potential role in the complex process of pain in knee OA (Cook 2016).

Musculoskeletal ultrasonography (US) is a relatively new imaging tool that is noninvasive, safe and relatively inexpensive and is able to create static as well as dynamic images. In addition, it has been shown to be more sensitive than clinical examination in picking up peri- and intra-articular soft tissue lesions (Bevers et al. 2014).

Objectives: To investigate the cause of pain in Knee OA by comparing sonographic and clinical findings in painful and non- painful osteoarthritic knee.

Methods: A cross-sectional case-control study carried out on fifty patients attending to Sohag University Hospitals rheumatology and rehabilitation outpatient clinic with Knee OA fulfilling ACR clinical criteria. They were divided into two groups. Group A (53) patients with knee pain (VAS $33 \mathrm{~mm}$ ) during physical activity once at least in the previous 3 days prior to inclusion. Another (42) patients without knee pain for at least 1 month prior to inclusion (VAS $0 \mathrm{~mm}$ ). All of the participants were subjected to the following:

- Full history (demographic data and personal history, detailed history of general health condition and chronic or current diseases).

Knee clinical examination (including varus deformity angle assessment)

- Sonographic evaluation: of Effusion, Synovial hypertrophy, Baker's cyst, Enthesitis, Power Doppler by SOLAR score, menisci protrusion, bursitis, sonographic signs of Gout or CPPD and scoring of the osteophytes and cartilage

Results: Our study showed that the painful OA group are more obese, more varus deformities, effusion, synovial hypertrophy, cartilage changes, and higher grading of osteophytes than the control group. On the other hand, Baker cyst and meniscal protrusion echogenic foci, double contour, erosions, meniscal and cartilage calcification showed a non-significant difference between the two groups. We included all of the demographic, clinical and sonographic factors in a univariate regression analysis, and this analysis showed that synovial hypertrophy, effusion, the degree of cartilage changes, the degree of osteophytes, bursitis, and weight may be possible risk factors for pain among OA patients.

Abstract AB0958 - Table 1. Multivariate logistic regression analysis of the possible independent risk factors for pain among painful OA group compared to the non painful OA group.

\begin{tabular}{|c|c|c|c|}
\hline Item & $\begin{array}{l}\text { Odd's } \\
\text { matio }\end{array}$ & $\begin{array}{c}\text { Clof } \mathrm{P} \\
\text { Odd's ralue }\end{array}$ & $\begin{array}{l}\text { Rank asa } \\
\text { possible risk } \\
\text { forpaill }\end{array}$ \\
\hline Weight & 1.078 & $\begin{array}{ll}0.126 \cdot & 0.110 \\
1.602 & \end{array}$ & 6 \\
\hline Effusion & 4.12 & $\begin{array}{ll}2.17 \% & 0.013 \\
24.422 & \end{array}$ & 3 \\
\hline Symorial & 7.230 & $0.74 \cdot \quad 0.089$ & $t$ \\
\hline lypetropty & & 70526 & \\
\hline $\begin{array}{c}\text { Degiree of cartilage } \\
\text { changes }\end{array}$ & 9,233 & $\begin{array}{l}2.354 \cdot \quad 0.002 ? \\
38.53 ?\end{array}$ & 1 \\
\hline Gemde of osteophtyes & 12.268 & $\begin{array}{ll}2.112 & 0.003 \\
71.283 & \end{array}$ & 2 \\
\hline Bursitis & 5.51 & $0.239 \cdot \quad 0.296$ & j \\
\hline
\end{tabular}

Abstract AB0958 - Figure 1
Conclusions: Cartilage degeneration, osteophytes, effusion, synovial hypertrophy, bursitis, and overweight respectively, are the leading causes of pain in knee $\mathrm{OA}$

Disclosure of Interest: None declared

DOI: 10.1136/annrheumdis-2018-eular.3971

\section{AB0959 \\ CONSUMPTION OF DAIRY PRODUCTS IN RELATION TO PRESENCE OF CLINICAL KNEE OSTEOARTHRITIS: THE MAASTRICHT STUDY}

K. Denissen ${ }^{1,2}, \underline{A}$. Boonen $^{2,3}$, J. Nielen ${ }^{1,3,4}$, A. Feitsma ${ }^{5}$, E. van den Heuvel ${ }^{5}$, P. Emans ${ }^{6}$, S. Sep ${ }^{7,8,9,10}$, C. Stehouwer ${ }^{7,10}$, M. van Dongen ${ }^{1,2}$, P. Dagnelie ${ }^{1,2,10}$ S. Eussen ${ }^{1,10}$. 'Department of Epidemiology, ${ }^{2} \mathrm{CAPHRI}$ Care and Public Health' Research Institute, Maastricht University; ${ }^{3}$ Department of Internal Medicine, Division of Rheumatology; ${ }^{4}$ Department of Clinical Pharmacy and Toxicology, Maastricht University Medical Center+, Maastricht, ${ }^{5}$ Friesland Campina, Amersfoort, ${ }^{6}$ Department of Orthopaedics; ${ }^{7}$ Department of Internal Medicine, Maastricht University Medical Center+; ${ }^{8}$ Department of Rehabilitation Medicine, Maastricht University, Maastricht; ${ }^{9} \mathrm{CAPHRI}$ Care and Public Health Research Institute, Maastricht University, Amersfoort, ${ }^{10} \mathrm{CARIM}$ School for Cardiovascular Diseases, Maastricht University, Maastricht, Netherlands

Background: Observational studies showed inverse associations between milk consumption and knee osteoarthritis (knee OA). ${ }^{1,2}$ There is lack of information on the role of specific other dairy product categories.

Objectives: To explore the association between dairy consumption and pres ence of clinical knee OA in individuals aged 40-75 year participating in the Maastricht Study.

Methods: Presence of clinical knee OA was defined according to a slightly modified version of the American College of Rheumatology (ACR) clinical classification criteria. ${ }^{3}$ Data on dairy consumption was appraised by a 253-item Food Frequency Questionnaire covering 47 dairy products with categorization on fat content, fermentation or dairy type. Multivariable logistic regression analyses were performed to estimate odd ratios (ORs) and 95\% confidence intervals $(95 \% \mathrm{Cl})$, while correcting for relevant factors.

Results: Of the 3010 participants included in this study, 427 individuals (14\%) were classified as having clinical knee OA. Significant inverse associations were observed between presence of clinical knee OA and intake of full-fat dairy and Dutch, primarily semi-hard, cheese, with OR for the highest compared to the lowest tertile of intake of $0.68(95 \% \mathrm{Cl} 0.50-0.92)$ for full-fat dairy, and $0.75(95 \% \mathrm{C}$ 0.56-0.99) for Dutch cheese. No significant associations were found for other dairy product categories.

Conclusions: In this Dutch population, higher intake of full-fat dairy and Dutch cheese, but not milk, was cross-sectionally associated with lower presence of clinical knee OA. Prospective studies need to assess the relationship between dairy consumption, and in particular semi-hard cheeses, with incident knee OA.

\section{REFERENCES :}

[1] Kacar C, et al. Clin Exp Rheumatol. 2004;22(4):473-6.

[2] Lu B, et al. Arthritis Care Res. 2014;66(6):802-9.

[3] Altman R, et al. Arthritis Rheum. 1986;29(8):1039-49.

Acknowledgements: The authors thank all the voluntary participants from the Maastricht Study as well as the funding bodies.

Disclosure of Interest: K. Denissen: None declared, A. Boonen: None declared J. Nielen: None declared, A. Feitsma Employee of: FrieslandCampina, a dairy company, E. van den Heuvel Employee of: FrieslandCampina, a dairy company, P. Emans: None declared, S. Sep: None declared, C. Stehouwer: None declared M. van Dongen: None declared, P. Dagnelie: None declared, S. Eussen: None declared

DOI: 10.1136/annrheumdis-2018-eular.4686

\section{AB0960 \\ POLYMORBIDITY AND COGNITION IN AMBULATORY POSTMENOPAUSAL HIP AND KNEE OSTEOARTHRITIS PATIENTS}

A.A. Popov ${ }^{1}$, N.V. Izmozherova ${ }^{2}$, N.A. Getmanova ${ }^{2} .{ }^{1}$ Hospital Therapy Dept; ${ }^{2}$ Pharmacology and Clinical Pharmacolgy Dept., Urals State Medical University, Ekaterinburg, Russian Federation

Background: Osteoarthritis $(O A)$.has been reported to be a risk factor of morbid ity, disability and premature cardiovascular mortality.

Objectives: to assess the impact of polymorbidity on cognitive function in postmenopausal women with primary $O A$. 